\title{
Sorafenib in metastatic radioactive iodine-refractory differentiated thyroid cancer: A pilot study
}

\author{
YANG LUO ${ }^{1,2}$, YUANKAI SHI ${ }^{1,2}$, PUYUAN XING ${ }^{1,2}$, LIN WANG $^{1,2}$, \\ YUN FENG ${ }^{1,2}$, XIAOHONG HAN ${ }^{1,2}$ and XIAOHUI HE $\mathrm{HE}^{1,2}$ \\ ${ }^{1}$ Department of Medical Oncology, Cancer Institute/Hospital, \\ Chinese Academy of Medical Sciences and Peking Union Medical College; \\ ${ }^{2}$ Beijing Key Laboratory of Clinical Study on Anticancer Molecular Target Drugs, Beijing 100021, P.R. China
}

Received June 2, 2013; Accepted September 11, 2013

DOI: $10.3892 /$ mco.2013.199

\begin{abstract}
The incidence and mortality of thyroid cancer are on the increase worldwide and the treatment options for progressive, radioactive iodine (RAI)-refractory metastatic differentiated thyroid cancer (DTC) patients are currently limited. Sorafenib is a multikinase inhibitor that targets several molecular signals, which are believed to be involved in the pathogenesis of DTC. In this study, we reported our experience with the off-label use of sorafenib in Chinese cancer patients. A total of 8 patients ( 7 with papillary and 1 with follicular thyroid cancer) were recruited in this study. The partial response (PR) rate was $50.0 \%$ and 5 of the 8 patients $(62.5 \%)$ achieved a durable response. The median progression-free survival (PFS) and overall survival (OS) were 40.1 and 55.0 weeks, respectively. Lung metastases were more sensitive to sorafenib compared to lymph nodes. The tumor marker response was not in accordance with the radiological response, although patients with tumor marker complete response (CR) exhibited a longer PFS and OS compared to those without a CR. Common adverse events (AEs) included palmar-plantar erythrodysesthesia, hypertension, diarrhea, weight loss and alopecia. Grade 4 AEs comprised hypocalcemia (1 patient) and elevated amylase levels (1 patient). A dose reduction was required in $62.5 \%$ of the patients. In conclusion, sorafenib exhibited a clinically relevant antitumor activity in patients with progressive metastatic RAI-refractory DTC, although the majority of the patients required a dose reduction due to intolerable toxicity.
\end{abstract}

Correspondence to: Dr Yuankai Shi, Department of Medical Oncology, Cancer Institute/Hospital, Chinese Academy of Medical Sciences and Peking Union Medical College; Beijing Key Laboratory of Clinical Study on Anticancer Molecular Target Drugs, 17 Panjiayuan Nanli, Chaoyang District, Beijing 100021, P.R. China E-mail: syuankaipumc@126.com

Key words: radioactive iodine-refractory, differentiated thyroid cancer, sorafenib, Chinese

\section{Introduction}

The incidence and mortality of thyroid cancer are increasing worldwide. Differentiated thyroid cancer (DTC), namely papillary thyroid carcinoma (PTC) and follicular thyroid carcinoma (FTC), comprise $~ 94 \%$ of these cases (1). Despite the majority of the DTC patients having indolent disease, $\sim 5-10 \%$ of these patients will eventually develop metastatic disease, no longer responding to radioiodine (RAI) therapy or thyroid-stimulating hormone (TSH) suppression and exhibiting a more aggressive course and short survival (2-4). Doxorubicin is the only currently available treatment option that has been approved by the Food and Drug Administration (FDA) for such patients, with a progression-free survival (PFS) of only 2 months and a median overall survival (OS) of 8 months; however, it is accompanied by severe myelosuppression and cumulative cardiotoxicity (5). Therefore, RAI-refractory DTC is a disease requiring novel therapeutic options exhibiting better efficacy and less toxicity.

Activating mutations in certain genes were previously reported to play a critical role in the development of DTC and the majority of DTCs may be due to single activating somatic mutations in one of three genes: BRAF mutations, RET/PTC rearrangements and Ras mutations (6-9). Sorafenib is a small-molecule multikinase inhibitor that targets several molecular signals which have been proven as potential therapeutic targets in DTC. Four phase II trials using sorafenib for metastatic thyroid cancer were recently published (10-13) and the promising preliminary results of those studies prompted the off-label use of commercially available sorafenib in patients with metastatic DTC refractory to RAI and TSH suppression. This study aimed to assess the clinical efficacy and safety of sorafenib treatment in Chinese patients with thyroid cancer.

\section{Patients and methods}

Patient details. The patients with metastatic DTC treated with sorafenib between January, 2011 and June, 2012 were entered into a retrospective database. Adult patients had histologically proven PTC or FTC and RAI-refractory disease, defined as a cumulative RAI dose of $\geq 600 \mathrm{mCi}$ or no-iodine uptake on a post-radioactive iodine scan. All patients had evidence of 
progressive disease (PD) within 3 months prior to the initiation of sorafenib under adequate TSH suppression $(<0.5 \mathrm{mU} / \mathrm{l})$. Patients who were treated with single-agent sorafenib and at least one follow-up imaging study to assess response after 2 months of therapy were included in this series.

Sorafenib treatment. Patients were treated with $400 \mathrm{mg}$ sorafenib (BAY 43-9006; Bayer HealthCare Pharmaceuticals, Montville, NJ, USA), administered orally twice a day. Dose reductions and treatment interruptions were allowed due to toxicity. All patients but one were administered sorafenib until PD or intolerable toxicity.

Study endpoints and evaluation of efficacy. The primary endpoints were the determination of response and PFS. The secondary study endpoints included adverse events (AEs), OS and the correlation between radiographical response and tumor marker response.

Computed tomography scans were performed prior to and every 2-3 months following treatment. The evaluation of efficacy used Response Evaluation Criteria In Solid Tumors, version 1.0. The target lesions (TLs) were defined as those that could be accurately measured in at least one dimension, with a longest diameter of $\geq 1 \mathrm{~cm}$. A durable response was defined as partial response (PR) and stable disease (SD) lasting $>6$ months. The PFS was defined as the time from the initiation of sorafenib administration to disease progression or death. The OS was defined as the time from the initiation of sorafenib to the time of death from any cause or the last follow-up.

Tumor marker evaluation. Serum thyroglobulin (TG) levels were measured at the baseline and at the same time, corresponding to each radiographic assessment, in all patients. A tumor marker complete response (CR) was defined as a decline to the normal range (including the normal range at baseline). A tumor marker PR was defined as a $>25 \%$ decline from baseline, but to levels higher than the upper limit of the normal range. $\mathrm{PD}$ was defined as a $>25 \%$ increase from baseline. SD was defined as any response between PR and PD.

Safety profile. Hematological and non-hematological toxicities associated with sorafenib were classified according to the revised National Cancer Institute Common Toxicity Criteria for Adverse Events, version 4.0.

Statistical methods. SPSS software, version 11.5 (SPSS Inc., Chicago, IL, USA) was used for statistical analysis. The Kaplan-Meier method was used to estimate the median PFS, the median OS and the 1- and 2-year survival rates. The comparisons between groups with different tumor marker responses were performed with the log-rank test. The $\chi^{2}$ test was used to assess the correlation between tumor marker response and radiographic efficacy. The response in different organs was compared using the two-independent-samples test.

\section{Results}

Clinical characteristics. A total of 8 patients were included in this series. The median patient age was 55 years (range,
43-67 years) and $62.5 \%$ of the patients were female. Seven of the patients $(87.5 \%)$ had a diagnosis of PTC and 1 patient (12.5\%) presented with FTC. The lung parenchyma was the most common metastatic location (87.5\%), followed by the lymph nodes (neck, mediastinal, hilar and inguinal nodes) (62.5\%), bone (37.5\%), soft tissue (37.5\%), renal parenchyma $(12.5 \%)$ and trachea $(12.5 \%)$. All patients presented with $>1$ location of metastatic disease. Seven patients received $>600 \mathrm{mCi}$ of RAI (range, 620-1,820 mCi) and 1 patient had TLs with no iodine-uptake on a post-RAI scan performed under conditions of a low iodine diet and adequate TSH elevation; thus, all patients were considered to have refractory disease. There was a significant difference in the TG baseline levels among the patients (range, $0.9->29,997.0 \mathrm{ng} / \mathrm{ml}$ ).

Response and survival. PR was achieved in 4 of the 8 patients $(50.0 \%)$ within the first 6 months of treatment; SD was observed in $3(37.5 \%)$ and PD in 1 patient (12.5\%), which consisted of the development of new lymph node lesions. No patients achieved a CR. A durable response was observed in 5 of the 8 patients $(62.5 \%)$. At the time of data analysis, the cumulative number of patients with PD was $4(50.0 \%)$ and they all succumbed to the disease. Of note, 1 patient with PTC had a visible nodule on their neck that had decreased in size by $32.6 \%$ at 4 weeks and had a PR verified by CT at 8 weeks; however, the patient underwent a dose reduction to $400 \mathrm{mg} / \mathrm{day}$ due to palmar-plantar erythrodysesthesia at 12 weeks, developed PD 4 months later and eventually succumbed to the disease (Fig. 1). Another female patient had achieved a PR at 6 months; however, the sorafenib dose was decreased to $200 \mathrm{mg} /$ day due to grade 4 hypocalcemia, the patient experienced disease progression 3 months later and succumbed to the disease shortly thereafter.

The median PFS was 40.1 weeks, with 1- and 2-year PFS rates of $49.6 \%$. The median OS was 55.0 weeks. The 1- and 2-year survival rates were 85.7 and $42.9 \%$, respectively.

Response by organ site. The response of TLs by organ site was assessed and the TLs of the lungs (median change, -30.7\%; range, -67 to $0 \%$ ) were shown to be more sensitive compared to those of the lymph nodes (median change, 6.3\%; range, -42 to $44 \%$ ) (Wilcoxon rank-sum test $\mathrm{P}=0.009$ ). Tumor regression in response to sorafenib may be observed in the lungs with simultaneous progression in the lymph nodes in the same patient (Fig. 2).

Tumor marker response. The tumor marker response was assessed in all patients. CR was oserved in 4 of the 8 patients $(50.0 \%)$, including 2 patients with TG levels within the normal range at baseline; PR was observed in 2 patients (25.0\%) and $\mathrm{SD}$ in the remaining 2 patients $(25.0 \%)$. The overall median PFS was not reached in the patients with tumor marker CR, although their PFS was significantly longer compared to that of the patients with tumor marker PR or SD (26.1 weeks, $\left.\chi^{2}=6.834, \mathrm{P}=0.009\right)$. There was no significant correlation between tumor marker response with radiological response using the Pearson's coefficient test $\left(\chi^{2}=0.000, P=1.000\right)$.

Treatment tolerability and AEs. The median duration of the treatment was 3-25 months. The treatment was discontinued 
A

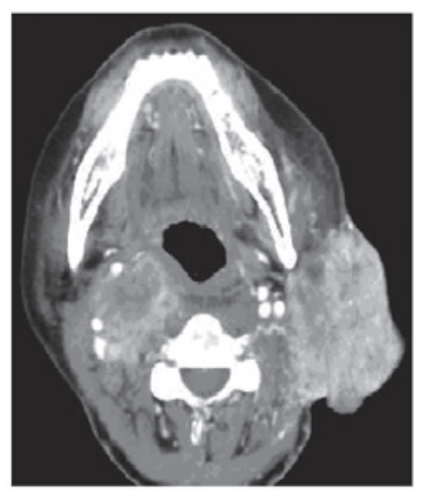

B

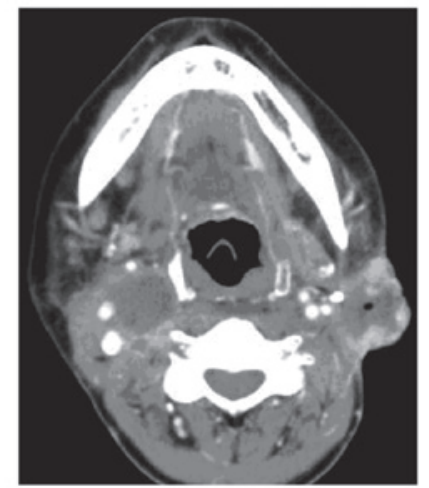

C

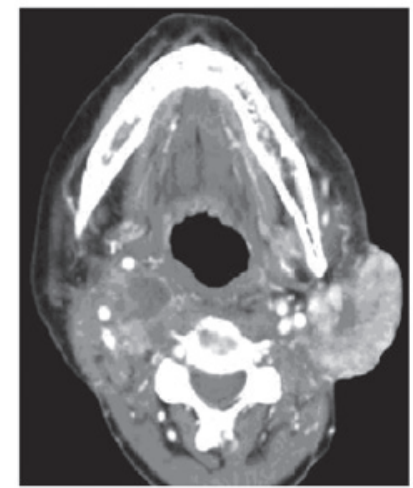

Figure 1. CT images of one partial response patient who experienced disease progression following a dose reduction of sorafenib. (A) Primary lesions prior to sorafenib treatment. (B) Primary lesion shrinkage after 4 months of sorafenib treatment; thereafter, sorafenib was reduced to $200 \mathrm{mg} / \mathrm{day}$, mainly due to toxicity. (C) Primary lesions showing progression 4 months later.

A

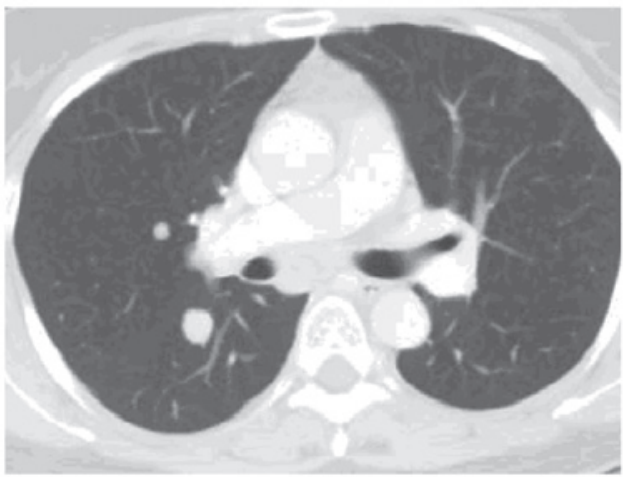

C

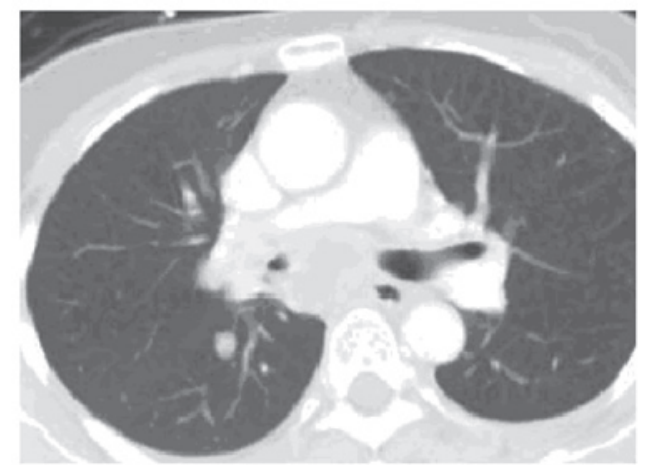

B

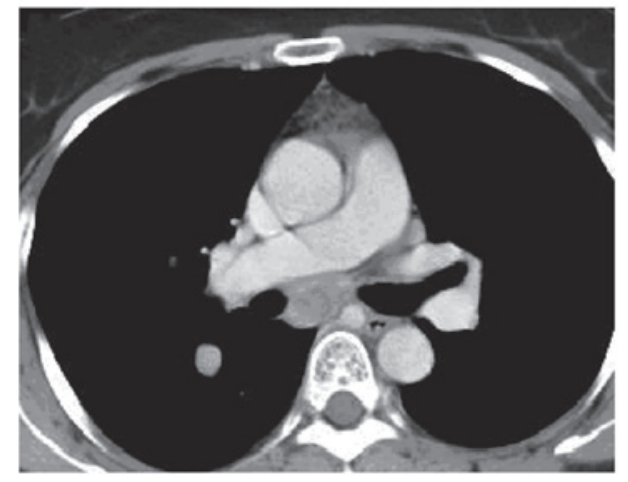

D

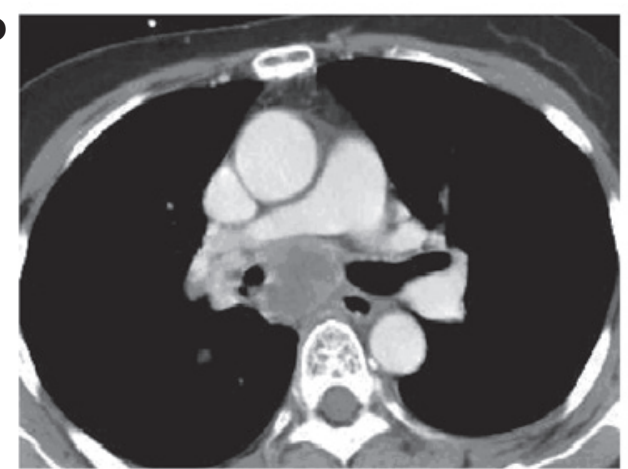

Figure 2. Partial response of the lung lesions with simultaneous progression in the lymph nodes in the same patient. CT scans prior to sorafenib treatment: (A) lung window and (B) mediastinal window. CT scans after 8 months of sorafenib treatment: (C) lung window and (D) mediastinal window.

in 4 patients (2 patients had PD, 1 patient was administered radiotherapy due to severe pain attributed to iliac bone metastasis and soft tissue invasion and 1 patient could no longer afford the cost of the treatment after 3 months). Five patients $(62.5 \%)$ underwent a reduction in the dose of sorafenib due to AEs ( 2 patients required a dose reduction by one dose level to $600 \mathrm{mg} / \mathrm{day}, 1$ patient underwent a further reduction to $400 \mathrm{mg} /$ day and another 2 patients to the lowest dose level of $200 \mathrm{mg} / \mathrm{day})$. The median time to dose reduction for all toxicities was 56 days (range, 20-82 days). The toxicities are summarized in Table I. Hematological toxicities were uncommon and of low degree. In terms of non-hematological toxicities, palmar-plantar erythrodysesthesia was the most common AE and over half of the patients complained of alopecia, hypertension, diarrhea, fatigue and weight loss.
Electrolyte abnormalities were uncommon, although 1 patient experienced grade 4 hypocalcemia despite daily calcium injections. The only grade 4 gastrointestinal toxicity observed was an elevation in amylase levels, which subsided following a dose reduction.

\section{Discussion}

Sorafenib has been approved by the FDA for the treatment of unresectable hepatocellular carcinoma and advanced renal cell carcinoma. Sorafenib inhibits the tyrosine kinase activities of the vascular endothelial growth factor receptor (VEGFR)-2 and -3 , platelet-derived growth factor receptor, FMS-like tyrosine kinase 3 and c-Kit, as well as certain intracellular serine/threonine kinases (such as, C-Raf and B-Raf) (14). The 
Table I. Treatment-related adverse events according to the Common Toxicity Criteria for Adverse Events (version 4.0).

\begin{tabular}{|c|c|c|c|c|c|c|c|c|}
\hline \multirow[b]{2}{*}{ Event } & \multicolumn{2}{|c|}{ Grade 1} & \multicolumn{2}{|c|}{ Grade 2} & \multicolumn{2}{|c|}{ Grade 3} & \multicolumn{2}{|c|}{ Grade 4} \\
\hline & Patient no. & $\%$ & Patient no. & $\%$ & Patient no. & $\%$ & Patient no. & $\%$ \\
\hline
\end{tabular}

Blood and lymphatic

system disorders

Anemia
Cardiovascular acute
coronary syndrome

2

coronary syndrome

Eye disorders

Blurred vision

Gastrointestinal disorders

Diarrhea
Nausea
Oral mucositis

General disorders and

administration site conditions

Fatigue

3

Investigations

ALT/AST increased

Lymphocytopenia

Serum amylase increased

Weight loss

Metabolism and

nutrition disorders

Anorexia
Hypocalcemia
Hypokalemia

Nervous system disorders

Headache

Respiratory, thoracic and mediastinal disorders

$$
\begin{aligned}
& \text { Bronchopulmonary } \\
& \text { hemorrhage }
\end{aligned}
$$$$
\text { Epistaxis }
$$

Skin and subcutaneous

\begin{tabular}{|c|c|c|c|c|c|c|c|c|}
\hline Alopecia & 2 & 25.0 & 3 & 37.5 & 1 & 12.5 & - & - \\
\hline $\begin{array}{l}\text { Palmar-plantar } \\
\text { erythrodysesthesia }\end{array}$ & 1 & 12.5 & 3 & 37.5 & 3 & 37.5 & - & - \\
\hline Rash & 1 & 12.5 & - & - & - & - & - & - \\
\hline \multicolumn{9}{|l|}{ Vascular disorders } \\
\hline Hypertension & 2 & 25.0 & 2 & 25.0 & 1 & 12.5 & - & - \\
\hline
\end{tabular}
tissue disorders

ALT, alanine aminotransferase; AST, aspartate aminotransferase.
1

1

12.5

12.5

12.5

12.5

37.5

$-$

37.5

3

$$
-
$$

1

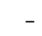

most common BRAF mutation is the V600E transversion, which may cause constitutive kinase activity and has been found in PTC (6). BRAF plays a critical role in cell signaling as an activator within the mitogen-activated protein kinase pathway. Gain-of-function mutations in the BRAF oncogene are the most frequent genetic alterations found in PTC, occurring in $\sim 45 \%$ of cases (8), as do Ras mutations (10\%) and rearrangements in the RET gene (RET/PTC) in 5-30\% of cases. These mutations were also described in FTC, affecting $>40 \%$ of cases and leading to a more aggressive disease (15). In addition, the overexpression of VEGF may contribute to the development and progression of DTC $(16,17)$. Those findings provided the rationale for using sorafenib in patients with metastatic DTC. 
The first trial of sorafenib in the treatment of metastatic thyroid cancer was reported by Gupta-Abramson et al (10). In that trial, 27 of 30 patients had DTC and of those patients, $68 \%$ had a diagnosis of PTC. Seven patients (26\%) had a PR lasting for 18-84 weeks, 16 patients (59\%) had SD lasting for 14-89 weeks and the median PFS was 79 weeks. Two phase II clinical trials of sorafenib reported an efficacy of this drug in metastatic DTC. In a study by Schneider et al (18), 31 patients with progressive, metastatic or locally advanced RAI-refractory DTC received sorafenib. After a median follow-up of 25 months, the response rates were $31 \%$ PR and $42 \%$ SD, the median PFS was 18 months and the median OS 34.5 months. In addition, Hoftijzer et al (12) reported a PR rate of $25 \%$, an SD rate of $34 \%$, a PD rate of $22 \%$ and a median PFS of 58 weeks in 31 patients with progressive DTC.

It was previously demonstrated that varying responses to treatment in different organs is often encountered in metastatic thyroid cancer patients treated with sorafenib. For example, lung metastases respond more favorably to sorafenib compared to lymph nodes (19). Cabanillas et al (20) reported the refractory nature of the bone metastases and pleural lesions. The pathophysiological mechanism underlying this variable response has not been fully elucidated. Whether these differences in response are due to the different expression of various VEGFRs or whether these are due to a non-VEGF-mediated mechanism, such as differences in drug concentrations among tissues, remains to be determined (19).

This pilot study presented our experience with metastatic, progressive RAI-refractory DTC treated with sorafenib at the Cancer Hospital of the Chinese Academy of Medical Sciences. Our results were a PR rate of $50 \%$, achieved within the first 6 months of treatment, with a durable response rate of $62.5 \%$. The median PFS and OS were 40.1 and 55.0 weeks, respectively. The PR rate appeared to be superior compared to that in published phase II trials evaluating sorafenib in DTC $(10-12,18)$. These differences in response may simply reflect the pre-study characteristics of our patient group. The majority of the patients (87.5\%) had developed lung metastases, which responded better to sorafenib compared to other organ lesions. However, inconsistent with the response rate, the PFS and OS were shorter compared to those observed in other phase II trials $(10,11,18)$. This difference may be attributed to the dose reduction due to the development of AEs, with 2 of the 3 patients who underwent a reduction in the dose of sorafenib to $<400 \mathrm{mg} /$ day experiencing disease progression and eventually succumbing to the disease. Our study also demonstrated that the lung metastases tended to respond better to sorafenib, with the lymph nodes being less sensitive. Tumor regression in response to sorafenib may occur in the lungs, with simultaneous lesion progression in the lymph nodes in the same patient. This variability of response by tissue site observed in these patients requires validation by larger studies.

Monitoring the disease with tumor markers may be of value in thyroid cancer. It is well known that serum TG is a highly sensitive and specific marker of DTC metastasis and recurrence and also reflects tumor burden. The change in serum TG levels prior to and following treatment was considered to be a prognostic indicator, assessing the efficacy of RAI treatment for bone metastases (21). However, our data suggested that the TG response in DTC patients treated with sorafenib did not correlate with radiological response $\left(\chi^{2}=0.000, P=1.000\right)$ and may not be relied upon as a marker of response. This result was in accordance with those reported in other phase II trials of tyrosine-kinase inhibitors (TKIs) in DTC, although the PFS and OS were longer in patients with tumor marker CR compared to those in patients without tumor marker CR (11). These results may reflect organ-specific tumor resistance, which is often encountered in patients with metastatic thyroid cancer treated with TKIs. Since DTC bone metastases may also produce and secrete TG, the serum TG levels are commonly significantly elevated in DTC patients with bone metastases. Sorafenib was shown to be less effective in patients with bone metastases (12). Notably, TLs were generally lung lesions which were more sensitive to sorafenib; therefore, the tumor marker response was not in accordance with the radiological response during TKI treatment for DTC, although patients with bone metastases with high levels of TG exhibited a significantly worse PFS and OS. This may suggest that, in patients with progressing bone lesions, TKI may not be effective and external beam radiation should be considered prior to TKI treatment.

The tolerability of sorafenib at the standard dose level was poor and $62.5 \%$ of the patients underwent a dose reduction due to AEs, with 2 patients having a dose reduction to the lowest level of $200 \mathrm{mg} / \mathrm{day}$. Palmar-plantar erythrodysesthesia was the most common AE, occurring in $87.5 \%$ patients and reaching grade 3 in $37.5 \%$ of the patients, followed by alopecia, hypertension, diarrhea, fatigue and weight loss. These results were similar to those reported by other phase II clinical trials of sorafenib in thyroid cancer $(11,13,18)$. Notably, 1 patient developed grade 4 hypocalcemia on sorafenib, despite daily intravenous calcium supplementation, and another patient experienced a grade 4 amylase level elevation and grade 3 weight loss and fatigue. All AEs ameliorated following a dose reduction of sorafenib to $200 \mathrm{mg}$ /day.

In conclusion, sorafenib appears to be of value in the treatment of patients with progressive metastatic RAI-refractory DTC, since it increases clinical response and prolongs PFS, even in patients exhibiting SD as their best response to treatment. However, the mechanism underlying the differential response of various metastatic sites (lungs, lymph nodes) requires further elucidation. Patients with bone metastases and high TG levels may be resistant to sorafenib and other prior treatment, such as radiotherapy, should be considered. However, more efficient agents or agent combinations are required to decrease the tumor burden and improve survival, without adversely affecting the quality of life of the patient.

\section{Acknowledgements}

This study was supported by Bayer Co., Ltd. and was partly funded by the Chinese National Major Project for New Drug Innovation (nos. 2008ZX09312 and 2012ZX09303012) and the Beijing Municipal Science and Technology Commission Major Project for New Drug Innovation (no. Z121107005112005), P.R. China.

\section{References}

1. Sherman SI: Thyroid carcinoma. Lancet 361: 501-511, 2003.

2. Mazzaferri EL: An overview of the management of papillary and follicular thyroid carcinoma. Thyroid 9: 421-427, 1999. 
3. Jonklaas J, Sarlis NJ, Litofsky D, et al: Outcomes of patients with differentiated thyroid carcinoma following initial therapy. Thyroid 16: 1229-1242, 2006.

4. Sampson E, Brierley JD, Le LW, et al: Clinical management and outcome of papillary and follicular (differentiated) thyroid cancer presenting with distant metastasis at diagnosis. Cancer 110: 1451-1456, 2007.

5. Shimaoka K, Schoenfeld DA, DeWys WD, et al: A randomized trial of doxorubicin versus doxorubicin plus cisplatin in patients with advanced thyroid carcinoma. Cancer 56: 2155-2160, 1985.

6. Caronia LM, Phay JE and Shah MH: Role of BRAF in thyroid oncogenesis. Clin Cancer Res 17: 7511-7517, 2011.

7. Cohen Y, Xing M, Mambo E, et al: BRAF mutation in papillary thyroid carcinoma. J Natl Cancer Inst 95: 625-627, 2003.

8. Henderson YC, Shellenberger TD, Williams MD, et al: High rate of BRAF and RET/PTC dual mutations associated with recurrent papillary thyroid carcinoma. Clin Cancer Res 15: 485-491, 2009.

9. Antonelli A, Fallahi P, Ferrari SM, et al: New targeted therapies for thyroid cancer. Curr Genomics 12: 626-631, 2011.

10. Gupta-Abramson V, Troxel AB, Nellore A, et al: Phase II trial of sorafenib in advanced thyroid cancer. J Clin Oncol 26 4714-4719, 2008

11. Kloos RT, Ringel MD, Knopp MV, et al: Phase II trial of sorafenib in metastatic thyroid cancer. J Clin Oncol 27: 1675-1684, 2009.

12. Hoftijzer H, Heemstra KA, Morreau H, et al: Beneficial effects of sorafenib on tumor progression, but not on radioiodine uptake, in patients with differentiated thyroid carcinoma. Eur J Endocrinol 161: 923-931, 2009.

13. Ahmed M, Barbachano Y, Riddell A, et al: Analysis of the efficacy and toxicity of sorafenib in thyroid cancer: a phase II study in a UK based population. Eur J Endocrinol 165: 315-322, 2011.
14. Wilhelm SM, Adnane L, Newell P, et al: Preclinical overview of sorafenib, a multikinase inhibitor that targets both Raf and VEGF and PDGF receptor tyrosine kinase signaling. Mol Cancer Ther 7: 3129-3140, 2008.

15. Garcia-Rostan G, Zhao H, Camp RL, et al: ras mutations are associated with aggressive tumor phenotypes and poor prognosis in thyroid cancer. J Clin Oncol 21: 3226-3235, 2003.

16. Vieira JM, Santos SC, Espadinha C, et al: Expression of vascular endothelial growth factor (VEGF) and its receptors in thyroid carcinomas of follicular origin: a potential autocrine loop. Eur J Endocrinol 153: 701-709, 2005.

17. Jo YS, Li S, Song JH, et al: Influence of the BRAF V600E mutation on expression of vascular endothelial growth factor in papillary thyroid cancer. J Clin Endocrinol Metab 91: 3667-3670, 2006.

18. Schneider TC, Abdulrahman RM, Corssmit EP, et al: Long-term analysis of the efficacy and tolerability of sorafenib in advanced radio-iodine refractory differentiated thyroid carcinoma: final results of a phase II trial. Eur J Endocrinol 167: 643-650, 2012.

19. Cabanillas ME, Waguespack SG, Bronstein Y, et al: Treatment with tyrosine kinase inhibitors for patients with differentiated thyroid cancer: the M. D. Anderson experience. J Clin Endocrinol Metab 95: 2588-2595, 2010.

20. Cabanillas ME, Hu MI, Durand JB, et al: Challenges associated with tyrosine kinase inhibitor therapy for metastatic thyroid cancer. J Thyroid Res 2011: 985780, 2011.

21. Qiu ZL, Song HJ, Xu YH, et al: Efficacy and survival analysis of ${ }^{131} \mathrm{I}$ therapy for bone metastases from differentiated thyroid cancer. J Clin Endocrinol Metab 96: 3078-3086, 2011. 